\title{
Effects of scopolamine on habituation of exploratory activity in rats
}

\author{
S. E. GREEN, DAPHNE JOYCE, and A. SUMMERFIELD \\ Birkbeck College, University of London, London, England WC1E $7 H X$
}

\begin{abstract}
Rats injected with scopolamine were more active than saline controls in a novel Y-maze. However, the habituation of locomotor activity over the 20-min trial was comparable for all groups. On a second trial 1 week later, with no injections, saline controls were less active, indicating a between-trial habituation of locomotor activity. This habituation was attenuated in all scopolamine groups, who were significantly more active on the second trial than saline controls. The results suggest a pharmacological differentiation of within- and between-trial habituation, and, in the absence of a drug effect on within-trial habituation, are interpreted in terms of memory rather than habituation processes. The between-trial effect was not obtained with the quaternary drug butylscopolamine. Finally, the dose-response curves for the increase in activity on Trial I and for the Trial II amnesic effect were found to be significantly different.
\end{abstract}

Carlton (1963, 1968, 1969; Carlton \& Markiewicz, 1971) has suggested that cholinergic pathways in the brain mediate the inhibition of nonreinforced responses. The decline in exploratory activity that occurs when a subject is repeatedly exposed to an initially novel environment may be considered a situation in which responses. no longer reinforced by novelty. are inhibited (Carlton. 1969). Such habituation of exploration should therefore be disrupted by injection of drugs which attenuate central cholinergic transmission.

To support this hypothesis. Carlton quotes from studies in his own laboratory (Carlton. 1969; Carlton \& Vogel. 1965) and from work done by Leaton (Leaton. 1968a. b. 1969). Carlton's procedure was to give rats a familiarization trial in a novel chamber. and then a second trial 2 days later in the same chamber; rats injected with the anticholinergic drug scopolamine before the first trial behaved on the second. undrugged. trial as if the chamber were again novel. Carlton concluded that the habituation which occurs in saline control animals on the first trial is disrupted in scopolamine animals. In Leaton's studies. rats were run in a T-maze. one arm of which contained novel objects; those injected with scopolamine made more choices of the novel "exploratory" arm when given repeated trials, as if the novelty of the objects was not habituating to the same degree as in control animals.

More recent studies indicate that the effects of scopolamine on exploratory responding may be more complex than originally thought. Walters and Block (1969) investigated both within- and between-trial habituation of exploration. and found that

At the time of this work. S.E.G. was in receipt of an M.R.C. Research Studentship. Address reprint requests to S.E.G., Department of Psychology. Birkbeck College. Malet Street. London WC1E ־HX. England. scopolamine while increasing overall levels of specific exploratory and general locomotor activity, did not affect their habituation. Graf (1974) also reports normal within-trial habituation of exploration in scopolamine-injected rats.

Feigley and Hamilton (1971) found a significant disruption of within-trial habituation of a nose-poke response in rats. However, the measure they used was response duration. which showed a steady increase over time in saline controls. Scopolamine-injected animals showed no increase. Response frequency was greater overall. but apparently habituated normally when compared with controls. Feigley and Hamilton did not discuss the relative importance of response frequency as opposed to response duration habituation.

In a replication of Carlton's original study (Carlton \& Vogel. 1965). Grant (1968) demonstrated an attenuation of both within- and between-trial habituation in mice injected with scopolamine on the first trial only. The degree of between-trial disruption was directly related to the degree of within-trial disruption.

There are thus positive and negative results in the literature. A source of some of the confusion lies in the various definitions of habituation used: although habituation within a single trial and habituation between two trials may be treated as aspects of a single phenomenon (Thompson \& Spencer, 1966). this may not be justitied (Groves \& Lynch, 1972). If not. experiments dealing with between-trial effects would not be directly comparable to those studying within-trial effects. Possibly those such as Grant's. which study both, are more fruitful.

The experiment to be reported investigates the effects of various doses of scopolamine on the exploratory activity of rats in a Y-maze. It was hypothesized that, on a first drugged trial, scopolamine would attenuate the decline in 
responding seen in control animals. It was further hypothesized that, on a second undrugged trial, scopolamine animals would be more active than controls, indicating a disruption of between-trial habituation.

\section{METHOD}

\section{Subjects}

The subjects were 72 naive male hooded rats of the Lister strain (Animal Suppliers, Ltd., Welwyn, Herts). They were housed four to a cage for 30 days and were 90 days old at the start of the experiment. The animals were maintained on ad-lib food and water throughout the experiment.

\begin{abstract}
Apparatus
Testing took place in a darkened room, with a background of white noise. The Y-maze was made of unpainted plywood, with arms 9 in. high, 5 in. wide, and 15 in. long. The floor was of gray cartridge paper, replaced after each animal. Illumination was provided by a $60-\mathrm{W}$ bulb suspended $15 \mathrm{in}$. above the center of the maze. Scoring was by observation.
\end{abstract}

\section{Injections}

Four doses of scopolamine hydrobromide were used: $0.25,0.50$, $1.0,2.0 \mathrm{mg} / \mathrm{kg}$. These were made up in normal saline. Control injections were of normal saline and of scopolamine butylbromide $(2.0 \mathrm{mg} / \mathrm{kg}$, made up in normal saline). Scopolamine butylbromide is a quaternary drug, and as such does not easily pass the blood-brain barrier; it was therefore used as a control for the peripheral effects of scopolamine.

All injections were IP, and of a volume calculated on the basis of $0.1 \mathrm{ml} / 100 \mathrm{~g}$ rat.

\section{Procedure}

The 72 subjects were split into six treatment groups. Four would receive varying doses of scopolamine hydrobromide, and two the control injections. There were 12 subjects in each group. Order of testing was randomized.

Each animal was removed from its home cage and injected $20 \mathrm{~min}$ before testing; during the injection-test interval, it was isolated. At the start of the test session, the subject was placed in

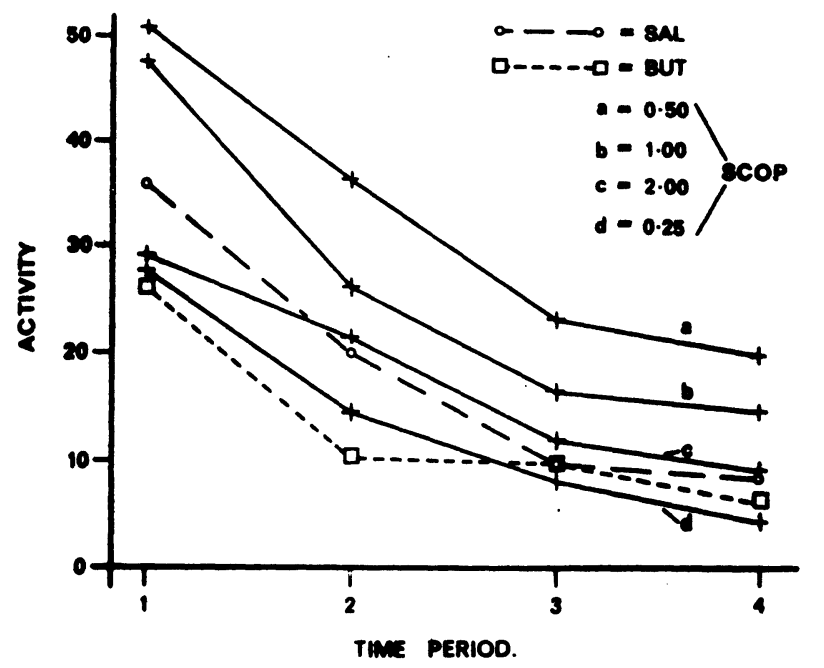

Figure 1. Mean frequency of locomotor activity over time for all groups, Trial I.
Table 1

Mean Overall Locomotor Activity for Each Group: Trial 1

\begin{tabular}{lc}
\hline Group & Activity \\
\hline SAL & 74.6 \\
BUT & $52.6^{*}$ \\
.25 SCOP & $54.3^{*}$ \\
.50 SCOP & $130.5^{*}$ \\
1.00 SCOP & $105.0^{*}$ \\
2.00 SCOP & 76.6 \\
\hline
\end{tabular}

*Comparison vs. SAL significant at $p<.01$.

the center of the maze, and its locomotor activity recorded for a 20 -min period. Scoring consisted of the number of arm entries (all four feet .entering an arm) combined with the number of arm traverses (all four feet passing a half-way marker on the wall of each arm). For the purposes of analysis, the 20-min session was split into four 5-min periods.

Trial II took place 7 days later. There were no injections. Otherwise the procedure was identical to Trial I.

\section{RESULTS}

Successive periods within a trial will be referred to as first, second, third, and fourth periods, respectively. Treatment groups will be referred to as follows: saline $=\mathrm{SAL}$, scopolamine butylbromide $=$ BUT, scopolamine $0.25 \mathrm{mg} / \mathrm{kg}=0.25 \mathrm{SCOP}$, scopolamine $0.50 \mathrm{mg} / \mathrm{kg}=0.50$ SCOP, scopolamine $1.00 \mathrm{mg} / \mathrm{kg}=1.00 \mathrm{SCOP}$, scopolamine $2.00 \mathrm{mg} / \mathrm{kg}$ $=2.00 \mathrm{SCOP}$.

All computations were based on Kirk (1968). Trial I data was analyzed using the appropriate split-plot design for one between factor (treatment) at six levels and one within-factor (time) at four levels. This was followed by a test of simple main effects.

All pairwise comparisons were made using Tukey's ratio, except for the comparison of each group's Trial I and Trial II scores. These were computed using correlated $t$ tests.

\section{Trial I}

The overall analysis indicated significant effects due to the treatment factor $(p<.01)$, the time factor $(p<.001)$, and their interaction $(p<.05)$. Tests of simple main effects revealed that differences due to treatment were significant only in the first two time periods $(\mathrm{p}<.001$ and $\mathrm{p}<.01$, respectively). Differences due to time were highly significant at all levels of treatment ( $p<.001$ in all cases).

Pairwise comparisons of each .group mean for Trial I with SAL indicated that the groups BUT and 0.25 SCOP were significantly less active overall ( $p<.01$ for both), groups 0.50 SCOP and 1.00 SCOP were more active ( $p<.01$ for both), and group 2.00 SCOP was not significantly different.

With reference to Figure 1, pairwise comparisons were made between each treatment group mean and SAL at every level of time. The 0.50 SCOP group was 
significantly more active during the first two time periods ( $p<.05$ in both cases). There were no other comparisons significant at the $\mathrm{p}<.05$ level.

$W$ ithin each treatment group. comparisons were made between activity scores for adjacent time periods. All groups showed a significant decline in activity between the first and the second periods $(p<.01$. except for 2.00 SCOP. where $p<.05)$. Except for the BUT group. all groups exhibited a significant drop in activity between the second and the third period $(\mathrm{p}<.01$. except for 0.25 SCOP, where $p<.05)$. The two scores for BUT were not signiticantly different.

There were no significant differences in any groups between the third and the fourth periods.

\section{Trial II}

Correlated $t$ tests were used to compare each group's overall activity score on Trial II with their score on Trial I.

SAL and BUT groups showed a significant drop in activity between the two trials $(\mathrm{p}<.001$ and $\mathrm{p}<.05$. respectively). as did the 0.50 SCOP and 1.00 SCOP groups $(\mathrm{p}<.01 . \mathrm{p}<.05)$. The Trial I and Trial II scores for the 0.25 and 2.00 SCOP groups were not signiticantly different.

Pairwise comparisons were made between each treatment group's overall activity score for Trial II and saline controls on Trial II. The BUT group was not significantly different from the SAL group, but all the SCOP groups were significantly more active $(p<.01$ in each case).

\section{Dose-Response Curves}

With reference to Figure 3, polynomial functions were titted to the two curres. log dose scopolamine

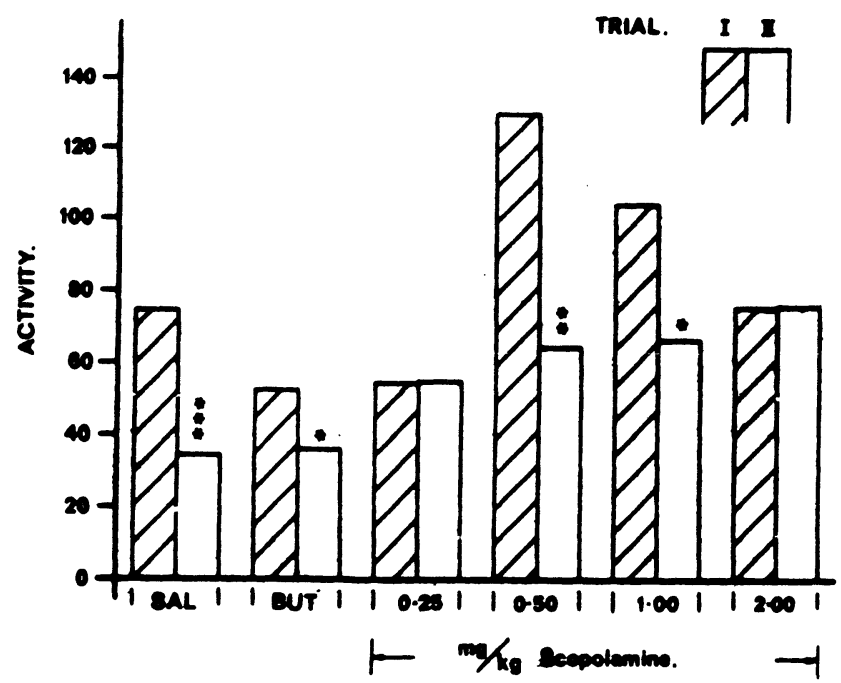

Figure 2. Mean overall locomotor activity for all groups, Trials I and II. Comparison of Trial I score with Trial II significant at: ${ }^{*} \mathrm{p}<.05 .{ }_{* \mathrm{p}}^{*}<.01,{ }_{*}^{*} \mathrm{p}<.001$.
Table 2

Mean Overall Locomotor Activity for Each Group: Trial 2

\begin{tabular}{ll}
\hline Group & Activity \\
\hline SAL & 34.3 \\
BUT & 36.4 \\
.25 SCOP & $54.8^{*}$ \\
.50 SCOP & $65.0^{*}$ \\
1.00 SCOP & $67.0^{*}$ \\
2.00 SCOP & $76.8^{*}$ \\
\hline
\end{tabular}

*Comparison vs. SAL significant at $p<.01$.

hydrobromide on Trial I plotted against activity on Trial I. and log dose scopolamine hydrobromide on Trial I plotted against activity on Trial II. For the former, there was a significant quadratic trend $(\mathrm{p}<.01)$, and for the latter. a significant linear trend $(\mathrm{p}<.05)$. No other components were significant at the $\mathrm{p}<.05$ level.

\section{DISCUSSION}

All groups showed a significant decline in locomotor activity on Trial I; this represents within-trial habituation of exploratory responding, elicited by exposure to an initially novel environment. There was a significant interaction between the treatment factor and the time factor; on the basis of pairwise comparisons, this may be attributed partly to the behavior of the BUT group, who did not demonstrate significant habituation after the second period. and partly to the tendency of the 0.50 SCOP group to be more active than saline controls early in the trial. As this latter finding would imply relatively faster habituation in this group. it does not suggest that scopolamine was attenuating within-trial habituation.

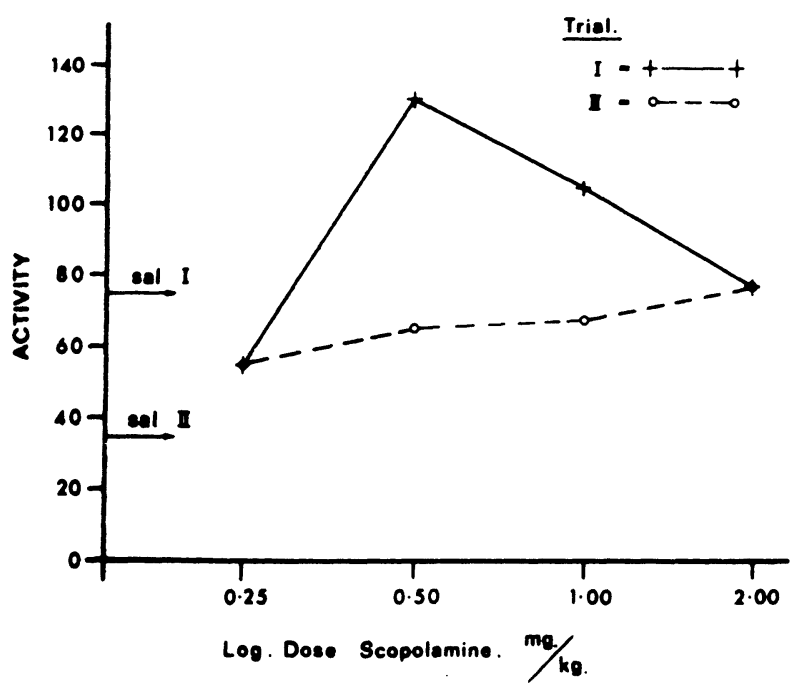

Figure 3. Dose-response curves. Log dose scopolamine on Trial I vs. activity on Trial I; log dose scopolamine on Trial I vs. activity on Trial II. 
The pattern of activity in the SCOP groups over time was comparable to that of the SAL group: a steady decline in locomotor activity between the first three time periods and a leveling off towards the end of the session. The more erratic behavior of the BUT group was probably due to the effects of the drug on peripheral cholinergic synapses.

Thus, the data from Trial I does not support the hypothesis that scopolamine attenuates the withintrial habituation of locomotor exploration. Overall activity was significantly increased in the 0.50 and 1.00 SCOP groups, but habituation was comparable to that seen in the SAL group. Support is therefore given to the findings of Walters and Block (1969) and Graf (1974).

The SAL group was significantly less active on the second, undrugged, trial, demonstrating the between-trial habituation of exploratory activity. The BUT group, despite their relatively depressed level of activity on Trial $I$, also showed a significant decline between trials, and on Trial II were not significantly different from the SAL group.

The 0.25 SCOP and 2.00 SCOP groups exhibited no drop in activity between trials, and on Trial II were significantly more active than the SAL group. The 0.50 SCOP and 1.00 SCOP groups did show a signiticant fall in activity between trials; however, that this fall was due to their elevated activity on Trial I is indicated by their being significantly more active on Trial II than the SAL group on Trial II. The finding that all SCOP groups were more active on Trial II than the SAL group suggests that they did not habituate to the same extent between trials. As Figure 2 indicates, they behaved on the second trial in a comparable manner to the SAL group on the first trial.

Thus, the data from Trial II helps to validate the hypothesis that injection of scopolamine attenuates the between-trial habituation of exploratory activity, so supporting the work of Carlton (1969) and Grant (1968). However, the absence of a drug effect on within-trial habituation points to a pharmacological differentiation of within- and between-trial habituation that considerably complicates any interpretation. Grant $(1968,1974)$ has presented data relating disruption of between-trial habituation to disruption of within-trial habituation; the present paper indicates that the two may be affected independently of each other. The conflicting results may be due to the different species or procedures involved.

A between-trials paradigm can lend itself to interpretations based on habituation phenomena or on memory phenomena (Carlton, 1969). It is difficult to operationally distinguish the two, as Trial II behavior used to assess habituation is dependent on the registration of the Trial I experience. Although Carlton points out that the effects he reports cannot be obtained with appetitive as opposed to aversive tasks-thus eliminating a general memorial interpretation of scopolamine effects-the idea of a cholinergic involvement in memory for "fearful" events has been proposed (Margules \& Margules, 1973). In the absence in the present experiment of a drug effect on within-trial habituation, it appears parsimonious to interpret the actions of scopolamine in terms of a memory rather than a habituation deficit; such an explanation would be consistent with the state-dependency effects which have been described for scopolamine (Berger \& Stein, 1969; Overton, Note 1).

Finally, the dose-response curves for the action of scopolamine on Trial I activity and for the Trial II amnesic effect are different. The amnesic effect is linearly related to dose, while the curve describing the stimulant action on activity is quadratic.

If the behavioral effects of scopolamine are directly linked to its pharmacological action as a competitive antagonist at cholinergic synapses (Brimblecombe, 1974), a linear dose-response curve would be predictable; however, a quadratic function would involve a more complex pharmacological model, and current work in this laboratory is aimed at possible bases for such a model.

\section{REFERENCE NOTE}

1. Overton, D. A. T-maze choice controlled by anticholinergic drugs; dose response studies. Paper read at 1969 Eastern Psychological Association Meeting, Philadelphia, Pennsylvania.

\section{REFERENCES}

Berger, B. D., \& Stein, L. An analysis of the learning deficits produced by scopolamine. Psychopharmacologia, 1969, 14, 271-283.

Brimblecombe, R. W. Drug actions on cholinergic systems. London: Macmillan Press, 1974.

Carlton, P. L. Cholinergic mechanisms in the control of behavior by the brain. Psychological Review, 1963, 70, 19-39.

Carlton, P. L. Brain-acetylcholine and habituation. Progress in brain research, 1968, 28, 48-60.

Carton, P. L. Brain-acetylcholine and inhibition. In J. T. Tapp (Ed.), Reinforcement and behavior. New York: Academic Press, 1969. Pp. 286-327.

Carlton, P. L., \& Markiewicz, B. Behavioral effects of atropine and scopolamine. In E. Furchtgott (Ed.), Pharmacological and biophysical agents and behavior. New York: Academic Press, 1971. Pp. 345-373.

Carlton, P. L., \& Vogel, J. R. Studies of the amnesic properties of scopolamine. Psychonomic Science, 1965, 3, 261-262.

Feigley, D. A., \& Hamilton, L. W. Response to novel environment following septal lesions or cholinergic blockade in rats. Journal of Comparative and Physiological Psychology, 1971, 76, 496-504.

GRAF, C. L. Effects of scopolamine on inhibitory mechanisms. Physiological Psychology, 1974, 2, 164-170.

GRANT, M. Effects of cholinergic drugs on habituation. Unpublished doctoral dissertation, University of Tennessee, 1968.

GRANT, M. Cholinergic influences on habituation of exploratory activity in mice. Journal of Comparative and Physiological Psychology, 1974, 86, 853-857. 
Groves. P. M.. \& LYNCH. G. S. Mechanisms of habituation in the brain stem. Psychological Review. 1972. 79. 237-244.

KIRK. R. E. Experimental design: Procedures for the behavioural sciences. Belmont. Calif: Brooks Cole. 1968.

LEATON. R. N. Effects of scopolamine on exploratory motivated behavior. Joumal of Comparative and Physiological Psychologi. 1908. 66. $524-52^{-}$. (a)

LEATON. R. N. Effects of scopolamine and eserine on position discrimination learning with an exploratory incentive. Psychonomic Science. 1968. 12. 181-182. (b)

LEATON. R. N. Effects of scopolamine and methscopolamine on brightness discrimination reinforced by opportunity for exploration. Journal of Comparative and Physiological Psychology. 1969. 68. $155-158$.

Marglies, D. L.. \& Margules. A. S. The development of operant responses by noradrenergic activation and cholinergic suppression of movements. In J. D. Maser (Ed.). Etfferent organisation and the integration of behavior. New York: Academic Press. 19:3. Pp. 203-230.

Thompson. R. F.. \& SPENCER. W. A. Habituation: A model phenomenon for the study of neuronal substrates of behavior. Psichological Review. 1966. 73. 16-43.

Walters, G.. \& Block. R. G. Scopolamine effects on locomotor and exploratory activity in rats. Psychonomic Science. 1969. 17. 3-4.

(Received for publication April 29. 1975; revision received June 3. 1975.) 